\title{
Akuter Ablationserfolg supraventrikulärer Tachykardien - Erfahrungen eines tertiären Zentrums
}

Özkartal, Tardu ; Haegeli, Laurent ; Wober, Thomas ; Krasniqi, Nazmi ; Saguner, Ardan M ; Breitenstein, Alexander ; Brunckhorst, Corinna ; Lüscher, Thomas F ; Duru, Firat ; Steffel, Jan

Posted at the Zurich Open Repository and Archive, University of Zurich ZORA URL: https://doi.org/10.5167/uzh-135299

Journal Article

Published Version

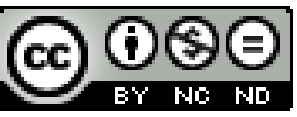

The following work is licensed under a Creative Commons: Attribution-NonCommercial-NoDerivatives 4.0 International (CC BY-NC-ND 4.0) License.

Originally published at:

Özkartal, Tardu; Haegeli, Laurent; Wober, Thomas; Krasniqi, Nazmi; Saguner, Ardan M; Breitenstein, Alexander; Brunckhorst, Corinna; Lüscher, Thomas F; Duru, Firat; Steffel, Jan (2016). Akuter Ablationserfolg supraventrikulärer Tachykardien - Erfahrungen eines tertiären Zentrums. Cardiovascular Medicine, 19(9):231-236. 


\section{Akuter Ablationserfolg supraventrikulärer Tachykardien}

Tardu Özkartal, Laurent Haegeli, Thomas Wolber, Nazmi Krasniqi, Ardan M. Saguner, Alexander Breitenstein, Corinna Brunckhorst, Thomas F. Lüscher, Firat Duru, Jan Steffel

Department of Cardiology, University Heart Center Zurich, Switzerland

\section{Summary}

Acute success rate for ablation of supraventicular tachycardia Experiences of a tertiary care center

Background: Catheter ablation is considered standard therapy for most patients with supraventricular tachycardia (SVT), with high success and low complication rates. This study sought to determine the frequency of ablation procedures from a Swiss tertiary care center, along with their acute success and complication rates.

Methods: All patients who had undergone electrophysiological testing for a suspected diagnosis of SVT at the University Heart Center of the University Hospital Zurich from 2012 to 2013 were included in this retrospective analysis.

Results: A total of 377 cardiac electrophysiological tests were performed for suspected SVT (mean patient age: $52 \pm 18$ years; $50 \%$ male). The most common SVT was atrioventricular nodal reentry tachycardia (AVNRT) in $48 \%$ ( $n=182$ ) of patients, followed by isthmus-dependent atrial flutter (IDAF) in $18 \%$ ( $n=69$ ), and manifest or concealed Wolff-Parkinson-White (WPW) in $17 \%(n=65)$. The acute success rate for the target ablation was $100 \%$ in both typical AVNRT ( $n=180$ out of 180 ) and IDAF ( $n=68$ out of 68 ). For treatment of WPW syndrome, $89 \%$ of patients ( $n=55$ out of 62 ) were successfully ablated. The acute success rate was $85 \%$ ( $n=17$ out of 20 ) for focal atrial tachycardia (FAT) and $73 \%$ ( $n=8$ out of 11 ) for atypical atrial flutter/intraatrial reentry-tachycardia (IART). Complications occurred at an incidence of $1.1 \%$. There were no peri-interventional deaths. Overall $43 \%$ of examinations were carried out in an outpatient setting.

Summary and conclusions: Our study results confirm that catheter ablation is effective and safe, supporting ablation therapy as a curative treatment for SVT management, which is often performed on an ambulatory basis. The success and complication rates from our tertiary center are comparable to those reported in the literature.

Key words: supraventricular tachycardia; AVNRT; SVT; WPW; Wolff-Parkinson-White-Syndrome; atrial tachycardia; atrial flutter

\section{Einleitung}

Supraventrikuläre Tachykardien (SVT) sind häufige Rhythmusstörungen. In einer epidemiologischen Studie aus den USA betrug die Prävalenz 2,3/1000 Personen und die Inzidenz 35/100000 Personenjahre [1]. Verschiedene Studien einzelner supraventrikulärer Tachykardien haben gezeigt, dass die interventionelle Katheterablation eine hohe Erfolgs- und eine geringe Komplikationsrate aufweist [1]. Eine medikamentöse Langzeitbehandlung ist hingegen - je nach Tachykardie - deutlich seltener erfolgreich [2]. Studien aus den 80er und 90er Jahren haben zudem gezeigt, dass Medikamente wie Verapamil [3], Flecainid [4], Propafenon [5] und Sotalol [6] zwar die Häufigkeit und Dauer der Episoden reduzieren, sie jedoch selten vollständig unterdrücken können. Ausserdem haben sie den Nachteil, dass sie meist dauerhaft eingenommen werden müssen (ausser bei einem "pill-in-the-pocket»-Vorgehen) und daher in der Langzeitanwendung häufig Nebenwirkungen verursachen.

Als Folge hat sich die Ablationsbehandlung in den letzten Jahrzehnten zunehmend zur Standardtherapie für die meisten supraventrikulären Tachykardien etabliert, mit entsprechender Klasse-I-Indikation in den aktuellen Guidelines [7]. Ziel der vorliegenden Arbeit war es, die Häufigkeitsverteilung der verschiedenen supraventrikulären Tachykardien sowie die akuten Erfolgs- und Komplikationsraten der Ablationsbehandlungen eines universitären Schweizer Zentrums zu analysieren und diese mit den internationalen Zahlen zu vergleichen.

\section{Methodik}

Studienpopulation und elektrophysiologische Untersuchungen

Sämtliche Patienten, die sich in den Jahren 2012 und 2013 aufgrund der Verdachtsdiagnose einer SVT im Universitären Herzzentrum Zürich einer elektrophysiologischen Untersuchung unterzogen, wurden in die 
Studie eingeschlossen (Patienten mit Vorhofflimmern wurden nicht berücksichtigt). Die Krankengeschichten wurden retrospektiv analysiert und, wenn nötig, mit externen Befunden ergänzt.

Die Untersuchungen wurden durch mindestens einen von sechs interventionellen Rhythmologen (FD, JS, CB, LH, TW, NK), in der Regel mit Assistenz mindestens eines Elektrophysiologie-Fellows, durchgeführt. Nach Lokalanästhesie mit Lidocain und Punktion der rechten Femoralvene wurden üblicherweise drei diagnostische Katheter (rechter Vorhof / Koronarsinus, His-Bündel und rechtsventrikulärer Apex) positioniert. In Ausnahmefällen wurde die Untersuchung in Intubationsnarkose durchgeführt. Zur Ablation einer linksseitigen Rhythmusstörung erfolgte eine transseptale Punktion und in einzelnen Fällen eine arterielle Punktion mit retrograd aortalem Zugang. Vor einer Ablationsbehandlung von isthmusabhängigem Vorhofflattern wurde in der Regel auf eine transösophageale Echokardiographie (TEE) zum Ausschluss intrakardialer Thromben verzichtet, wenn zum Zeitpunkt der Ablation ein Sinusrhythmus vorlag oder eine suffiziente orale Antikoagulation während der vorangegangenen 4 Wochen bestand. Bei den übrigen Patienten, insbesondere vor Ablation linksseitiger atrialer Tachykardien oder eines atypischen Vorhofflatterns, erfolgte in der Regel vorgängig eine TEE.

Im Rahmen der intrakardialen Diagnostik wurden die Basisintervalle (AH- und HV-Zeit, AV-block cycle length (AVBCL), ERP, AH-Sprung, VA-Zeit usw.) gemessen. Mittels standardisierter atrialer und ventrikulärer Stimulationsprotokolle wurde anschliessend angestrebt, die Rhythmusstörung zu induzieren. Bei fehlender Auslösbarkeit wurde zusätzlich eine adrenerge Stimulation mittels Isoprenalin (1-6 $\mu \mathrm{g} /$ Minute) \pm Atropin (0,5-1 mg als Bolus) durchgeführt. Je nach Diagnose erfolgte bei gegebener Indikation im Rahmen derselben Untersuchung eine Radiofrequenz- und in ausgewählten Fällen (z.B. Nähe der Ablationsstelle zum Sinusknoten oder His-Bündel) eine Kryoablation.

Zur Ablation fokaler atrialer Tachykardien und atypischen Vorhofflatterns, sowie gelegentlich bei isthmusabhängigem VHFla und WPW, kam ein dreidimensionales Mapping-System, meist CARTO (Biosense Webster, Diamond Bar, CA), zum Einsatz.

Allgemein wurde die Behandlung als erfolgreich definiert, wenn die Rhythmusstörung im Anschluss an die Ablation (ggf. unter adrenerger Stimulation mit Isoprenalin) nicht mehr induziert werden konnte. Die Wartezeit nach Ablation betrug in der Regel 20-30 Minuten. Bei offenem Wolff-Parkinson-White-Syndrom galt das anhaltende Verschwinden der Delta-Welle (und Normalisierung der HV-Zeit sowie retrograde dekremen- tale konzentrische Erregung bzw. VA-Dissoziation) als Erfolgskriterium. Bei verborgenem WPW wurde eine Ablation bei anhaltendem Nachweis eines VA-Blocks als erfolgreich angesehen. Bei isthmusabhängigem Vorhofflattern galten eine Transisthmuszeit $>100 \mathrm{~ms}$, das Auftreten von Doppelpotentialen mit konstantem Intervall entlang der Ablationslinie sowie der Nachweis einer kranio-kaudalen Aktivierung der lateralen rechten Vorhofwand bei Stimulation aus dem proximalen Koronarsinus bzw. gegenläufiger Aktivierung während Stimulation mit dem Ablationskatheter lateral der Ablationslinie als Erfolgskriterium (bidirektionaler Block). Bei atrialen Tachykardien und atypischem Vorhofflattern wurde in der Regel eine Verlangsamung bzw. Verlängerung der Zykluslänge vor Terminierung der Tachykardie während der Ablation sowie eine anschliessende Nichtinduzierbarkeit trotz aggressiver Stimulation als Erfolg gewertet.

\section{Statistik}

Sämtliche statistischen Analysen wurden mit Microsoft Excel 2010 durchgeführt. Ein p-Wert <0.05 wurde als signifikant angesehen. Kategorische Variablen sind als (n/total) oder als \% (n) dargestellt, kontinuierliche als Mittelwert (+/- Standardabweichung).

\section{Resultate}

\section{Baseline-Charakteristika}

Die demographischen und klinischen Charakteristika der Patienten sind in Tabelle 1 aufgeführt. Insgesamt wurden etwa gleich viele Männer wie Frauen untersucht. Das durchschnittliche Alter betrug $52 \pm 18$ Jahre. Ungefähr ein Viertel der Patienten hatte eine bekannte Herzerkrankung.

Die häufigsten kardialen Grunderkrankungen, sofern vorhanden, waren eine koronare Herzerkrankung in $31 \%(n=27$ von 86$)$, ein angeborener Herzfehler sowie ein Klappendefekt in je 21\% (jeweils $\mathrm{n}=18$ von 86 ). Knapp ein Drittel (30\%; $\mathrm{n}=111$ von 372) der Patienten hatte eine arterielle Hypertonie und 3,2\% ( $n=12$ von 370) wiesen ein zerebrovaskuläres Ereignis in der Vorgeschichte auf.

Zur antiarrhythmischen Behandlung vor der elektrophysiologischen Untersuchung wurden am häufigsten Beta-Blocker verwendet (39\%; $n=146$ von 374), gefolgt von Amiodaron (5,6\%; $\mathrm{n}=21$ von 374 ) und Flecainid (2,7\%; $n=10$ von 374$)$. 86 Patienten nahmen Thrombozytenhemmer ein, zumeist Acetylsalicylsäure (87\%; n = 75 von 86). 79 Patienten wurden antikoaguliert, meist mit einem Vitamin-K-Antagonisten (66\%; $n=52$ von 79) und die übrigen mit Rivaroxaban. 
Tabelle 1: Baseline-Charakteristika der untersuchten Patienten.

\begin{tabular}{|c|c|}
\hline \multicolumn{2}{|l|}{ Demographische Daten } \\
\hline Alter (Jahre) & $52,1 \pm 18,4$ \\
\hline Männlich & $49,9(188 / 377)$ \\
\hline Grösse (m) & $1,70 \pm 0,1$ \\
\hline Gewicht (kg) & $75,4 \pm 18$ \\
\hline BMI $\left(\mathrm{kg} / \mathrm{m}^{2}\right)$ & $26 \pm 5,1$ \\
\hline \multicolumn{2}{|l|}{ Krankengeschichte } \\
\hline Herzfrequenz (bpm) im Sinusrhythmus & $78 \pm 18$ \\
\hline Arterielle Hypertonie & $29,8(111 / 372)$ \\
\hline Diabetes mellitus & $8,1(30 / 372)$ \\
\hline Schlaganfall oder TIA in der Anamnese & $3,2(12 / 370)$ \\
\hline Kardiopathien & $23(86 / 374)$ \\
\hline Koronare Herzerkrankung & $31,4(27 / 86)$ \\
\hline Dilatative Kardiomyopathie & $4,7(4 / 86)$ \\
\hline Restriktive Kardiomyopathie & $3,5(3 / 86)$ \\
\hline $\begin{array}{l}\text { Arrhythmogene rechtsventrikuläre } \\
\text { Kardiomyopathie/Dysplasie }\end{array}$ & $1,2(1 / 86)$ \\
\hline Valvuläre Kardiomyopathie & $20,9(18 / 86)$ \\
\hline Kongenitales Vitium & $20.9(18 / 86)$ \\
\hline Hypertrophe Kardiomyopathie & $2,3(2 / 86)$ \\
\hline Andere & $15,1(13 / 86)$ \\
\hline \multicolumn{2}{|l|}{ Baseline-Medikamente $(n=374)$} \\
\hline Thrombozytenaggregationshemmer & $23(86 / 374)$ \\
\hline Acetylsalicylsäure & $87,2(75 / 86)$ \\
\hline Clopidogrel & $5,8(5 / 86)$ \\
\hline Prasugrel & $5,8(5 / 86)$ \\
\hline Ticagrelor & $1,2(1 / 86)$ \\
\hline Orale Antikoagulantien & $21,1(79 / 374)$ \\
\hline Vitamin-K-Antagonisten & $65,8(52 / 79)$ \\
\hline Rivaroxaban & $34,2(27 / 79)$ \\
\hline Betablocker & $39(146 / 374)$ \\
\hline Kalzium-Antagonist & $11(41 / 374)$ \\
\hline Amiodaron & $5,6(21 / 374)$ \\
\hline Flecainid & $2,7(10 / 374)$ \\
\hline Digoxin & $0,5(2 / 374)$ \\
\hline
\end{tabular}

Kategorische Variablen sind als \% (n/total) dargestellt, kontinuierliche als Mittelwert +/- Standardabweichung.

\section{EKG und Echokardiographie-Befunde vor der Ablation}

Bei den meisten Patienten lag als Grundrhythmus ein Sinusrhythmus vor (86\%; $\mathrm{n}=307$ von 359). Eine unmittelbar präinterventionelle Echokardiographie wurde bei 68\% ( $n=255$ von 377) Patienten durchgeführt (Tab. 2), meist bei Vorliegen einer fokalen atrialen Tachykardie oder von Tachykardien im Rahmen von kongenitalen Vitien. Die durchschnittliche linksventrikuläre Auswurffraktion der untersuchten Patienten betrug $60 \pm 9 \%$. Eine diastolische Dysfunktion konnte bei $32 \%$ ( $\mathrm{n}=46$ der 142 hierfür untersuchten Patienten) dokumentiert werden.
Tabelle 2: Echokardiographie-Befunde.

\begin{tabular}{|c|c|}
\hline \multicolumn{2}{|l|}{ Linksventrikuläre Parameter } \\
\hline Ejektionsfraktion (\%) & $59,5 \pm 9,2$ \\
\hline Enddiastolisches Volumen (indexiert) $\left(\mathrm{ml} / \mathrm{m}^{2}\right)$ & $52,8 \pm 15,9$ \\
\hline Enddiastolischer Diameter (mm) & $4,8 \pm 0,6$ \\
\hline \multicolumn{2}{|l|}{ Mitralinsuffizienz $(n=217)$ / linker Vorhof } \\
\hline Keine & $74,2(161)$ \\
\hline Leichtgradig & $22,6(49)$ \\
\hline Mittelschwer & $2,8(6)$ \\
\hline Schwer & $0,5(1)$ \\
\hline Linksatrialer endsystolischer Diameter ( $\mathrm{mm})$ & $3,7 \pm 0,8$ \\
\hline Linksatriales Volumen (indexiert) $\left(\mathrm{ml} / \mathrm{m}^{2}\right)$ & $33,3 \pm 13,3$ \\
\hline \multicolumn{2}{|l|}{ Rechtsherz-Funktion und Anatomie } \\
\hline RV-Fläche $\left(\mathrm{cm}^{2}\right)$ & $19,9 \pm 9,7$ \\
\hline Systolischer RV-Druck über RA-Druck (mmHg) & $22,2 \pm 7,6$ \\
\hline TAPSE (mm) & $21,8 \pm 5,2$ \\
\hline Flächenverkürzungsfraktion (fac) des RV (\%) & $39,5 \pm 11,8$ \\
\hline RA-Diameter (cm) & $4,8 \pm 1$ \\
\hline \multicolumn{2}{|l|}{ Diastolische Dysfunktion ( $n=142$ ) } \\
\hline Keine & $67,6(96)$ \\
\hline Relaxationsstörung & $25,4(36)$ \\
\hline Pseudonormalisierung & $4,2(6)$ \\
\hline Restriktion & $2,8(4)$ \\
\hline
\end{tabular}

\section{Häufigkeit der verschiedenen SVT und akuter Ablationserfolg}

Insgesamt wurden 377 elektrophysiologische Untersuchungen für SVTs durchgeführt, wobei in 92\% ( $\mathrm{n}=347)$ in der gleichen Sitzung eine Ablation erfolgte. Die akute Erfolgsrate aller abladierten supraventrikulären Tachykardien lag bei 96\% (334/347).

Die häufigste supraventrikuläre Rhythmusstörung war mit 48\% ( $\mathrm{n}=182)$ die typische AV-Knoten-ReentryTachykardie (AVNRT), welche in 100\% ( $n=180$ ) erfolgreich behandelt werden konnte (Tab. 3). Die zweithäufigste SVT war mit einem Anteil von 18\% ( $\mathrm{n}=69)$ das isthmusabhängige Vorhofflattern (VHFla), welches ebenfalls mit einer akuten Erfolgsrate von 100\% ( $n=68$ ) abladiert wurde. Die seltenste SVT war mit 1,6\% $(\mathrm{n}=6)$ die atypische AVNRT, welche in 100\% der Fälle erfolgreich behandelt werden konnte. Das Wolff-Parkinson-White-Syndrom lag mit $17 \%(\mathrm{n}=65)$ an dritter Stelle und konnte in $89 \%$ der Fälle ( $n=55$ von 62 ) erfolgreich interventionell therapiert werden. Bei den erfolglosen Ablationen war die Bahn in zwei Fällen parahissär lokalisiert, so dass nach kurzem Mapping und wenigen kurzen Ablationsversuchen ein weiteres Vorgehen mit Radiofrequenz nicht vertretbar erschien. Ein weiterer Patient hatte mehrere, teils epikardial ver- 
Tabelle 3: Prävalenz der supraventrikulären Tachykardien.

\begin{tabular}{|c|c|}
\hline Typische AVNRT & $48,3(182)$ \\
\hline Typisches Vorhofflattern & $18,3(69)$ \\
\hline WPW-Syndrom/AVRT & $17,2(65)$ \\
\hline Fokale atriale Tachykardie & $10,6(40)$ \\
\hline Atypisches Vorhofflattern/IART & $4(15)$ \\
\hline Atypische AVNRT & $1,6(6)$ \\
\hline Gesamt & $100(377)$ \\
\hline
\end{tabular}

laufende Bahnen. Bei den übrigen vier Patienten war die Ablation auch nach mehrfacher Energieabgabe nicht erfolgreich, so dass bei beginnender Ödembildung der Eingriff beendet wurde. Hierbei ist zu erwähnen, dass bei zwei der initial erfolglos behandelten $\mathrm{Pa}$ tienten in einer zweiten Untersuchung, ausserhalb der analysierten Periode, die akzessorische Bahn erfolgreich abladiert wurde. Die geringsten Erfolgsraten la-

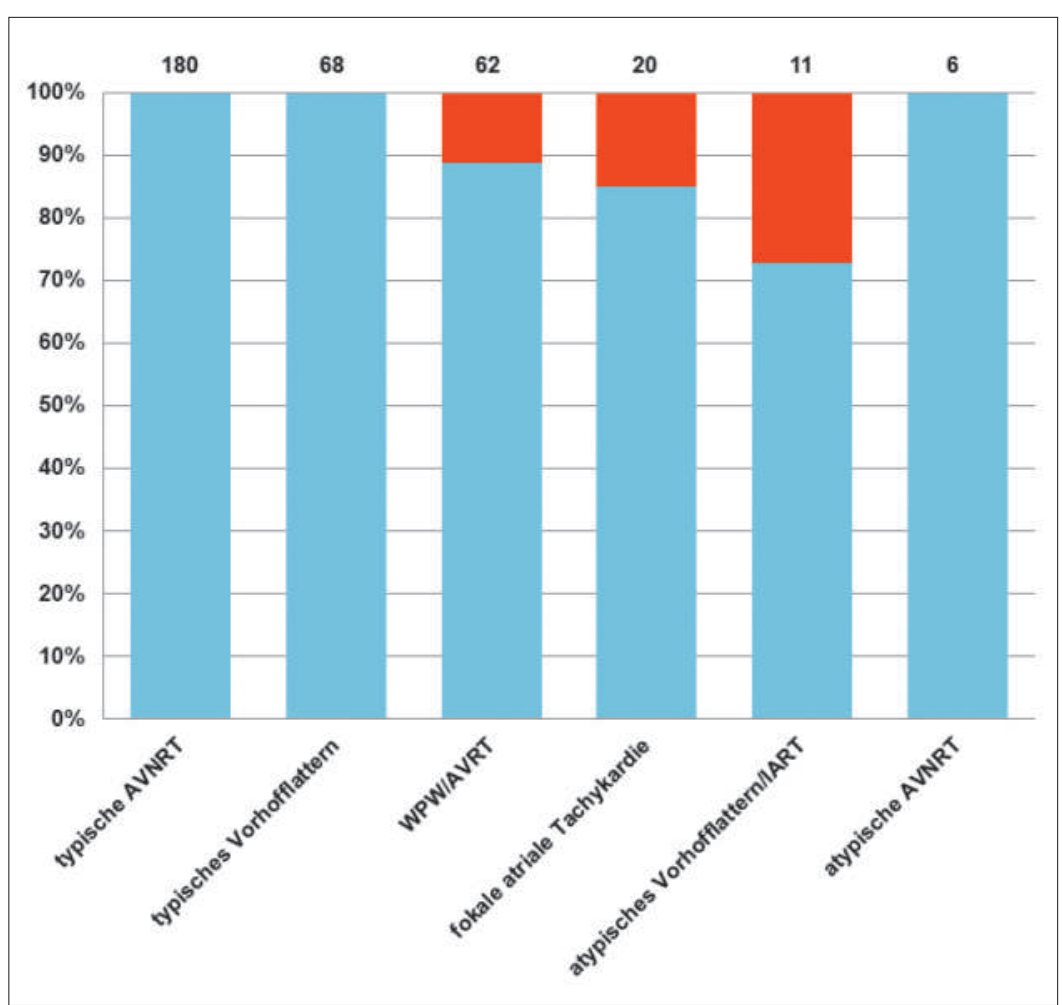

Abbildung 1: Akute Erfolgsrate bei Ablation. Dargestellt ist die absolute Zahl an Ablationsbehandlungen aufgeteilt nach supraventrikulärer Rhythmusstörung (blau - erfolgreich, rot - nicht erfolgreich).

AVNRT: Atrioventrikularknoten-Reentry-Tachykardie; WPW: Wolff-Parkinson-WhiteSyndrom: AVRT: atrioventrikuläre Reentry-Tachykardie. IART: intraatriale Reentry-Tachykardie. gen mit 85\% ( $\mathrm{n}=17$ von 20 ) bei fokalen atrialen Tachykardien (FAT), sowie mit $73 \%$ ( $\mathrm{n}=8$ von 11 Ablationen) bei atypischem Vorhofflattern (aVHFla) bzw. intraatrialen Reentry-Tachykardien (IART) vor (Abb. 1). Bei 8\% (n = 30 von 377) wurde a priori keine Ablation durchgeführt (AVNRT 2, typisches VHFla 1, WPW/AVRT 3, FAT 20, aVHFla/IART 4). Gründe hierfür waren, dass entweder (1) in 33\% keine anhaltende Rhythmusstörung induziert werden konnte ( $\mathrm{n}=10$ von 30 ), oder (2) in $23 \%$ eine Ablation bei Vorliegen multipler Foci von fokalen atrialen Tachykardien und/oder Vorliegen verschiedener Reentry-Kreise etc. wenig erfolgversprechend erschien ( $n=7$ von 30) oder (3) bei $20 \%$ aufgrund der anatomischen Lage die Gefahr einer Schädigung des AV- bzw. Sinusknotens bestand ( $\mathrm{n}=6$ von 30). Weitere, teilweise parallel vorliegende Gründe waren schwierige anatomische Verhältnisse sowie relativ oligosymptomatische Patienten mit akzeptablem Ansprechen auf eine medikamentöse Therapie.

\section{Ambulante versus stationäre Eingriffe}

Die Anzahl Hospitalisationstage ist in Abbildung 2 aufgeführt. Bei einem Grossteil der Patienten (78\%; n = 293 von 377 ) konnte die Untersuchung ambulant (43\%; $n=$ 163 von 377) oder im Rahmen einer Kurzhospitalisation mit einer Übernachtung (35\%; $\mathrm{n}=130$ von 377 ) durchgeführt werden. Bei lediglich 6,9\% ( $n=26$ von 377 ) der Patienten war eine Hospitalisation von mehr als 2 Nächten erforderlich, wobei sich diese häufig aufgrund einer anderen Ursache bereits in stationärer Behandlung befanden (z.B. elektive Operation, kardiale Dekompensation, u.a.m.) und die supraventrikuläre Tachykardie nebenbefundlich diagnostiziert und behandelt wurde.

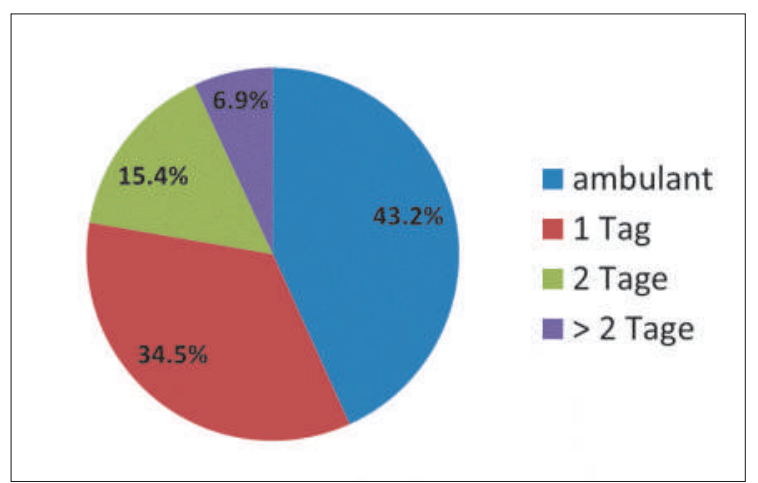

Abbildung 2: Hospitalisationstage. Dargestellt ist die prozentuale Verteilung der Patienten ( $n=377$ ) auf die Hospitalisationsdauer. Dabei entspricht 1 Tag einer Kurzhospitalisation mit einer Übernachtung, 2 Tage einer Hospitalisation mit 2 Übernachtungen und $>2$ Tage einer Hospitalisation mit mehr als zwei Übernachtungen. 


\section{Komplikationen}

Schwerwiegende Komplikationen traten bei vier Untersuchungen (1,1\%) auf. Während der Ablation eines slow-pathway des AV-Knotens aufgrund einer typischen AVNRT kam es bei 2 Patienten (1,1\% aller AVNRTAblationen) zu einer persistierenden kompletten AVBlockierung. Einer der Patienten blieb während sowie nach dem Eingriff aufgrund eines hohen Ersatzrhythmus oligosymptomatisch, so dass bis dato insbesondere auf Wunsch des Patienten, von einer Schrittmacher-Implantation abgesehen wurde. Beim zweiten Patienten hingegen musste ein Zweikammerschrittmacher implantiert werden (0,56\% der AVNRT-Ablationen bzw. 0,29\% aller Ablationen für SVT).

Bei einem Patienten mit isthmusabhängigem Vorhofflattern kam es postinterventionell zu einem grossen Hämatom in der rechten Leiste im Bereich der Punktionsstelle, was die Gabe von zwei Erythrozytenkonzentraten erforderlich machte. Der Patient stand unter einer Behandlung mit einem Vitamin-K-Antagonisten und der INR betrug zum Zeitpunkt der elektrophysiologischen Untersuchung 2,6. Das Hämatom musste aufgrund einer Superinfektion im Verlauf operativ ausgeräumt werden und heilte schliesslich folgenlos ab. Bei einem Patienten konnte postinterventionell nach linksseitiger WPW-Ablation echokardiographisch ein kleiner, hämodynamisch nicht relevanter Perikarderguss festgestellt werden, welcher keine therapeutischen Konsequenzen nach sich zog.

Periinterventionelle Todesfälle traten nicht auf.

\section{Diskussion}

Bei der vorliegenden Studie handelt es sich unserem Wissen nach um die erste aktuelle Zusammenstellung supraventrikulärer Tachykardien sowie der akuten Erfolgs- und Komplikationsraten von Ablationsbehandlungen eines universitären Zentrums in der Schweiz. Es zeigten sich verschiedene interessante Ergebnisse.

\section{Prävalenz supraventrikulärer Tachykardien}

In einer grossen Registerstudie aus Spanien [8] mit insgesamt 7659 Patienten war die AVNRT mit 37\% die am häufigsten interventionell therapierte supraventrikuläre Tachykardie, gefolgt von typischem Vorhofflattern mit 32\%. Am seltensten wurde mit 4,3\% der Fälle die fokale atriale Tachykardie behandelt. Auch in unserer Studienpopulation war die typische AVNRT mit $48 \%$ die am häufigsten abladierte SVT, gefolgt von isthmusabhängigem Vorhofflattern mit 18\%. Am seltensten wurden in unserer Kohorte atypisches, nicht-isthmusabhängiges Vorhofflattern $(4,0 \%)$ und die atypische
AVNRT $(1,6 \%)$ behandelt, wobei auch fokale atriale Tachykardien mit 5,6\% eher selten waren.

\section{Akuter Ablationserfolg}

In derselben Registerstudie aus Spanien [8] wurde die AVNRT in 98\% der Fälle erfolgreich abladiert, das Vorhofflattern in 97\% und das WPW-Syndrom in 91\%. Die geringste Erfolgsrate bestand mit $85 \%$ bei der fokalen atrialen Tachykardie. In einer Meta-Analyse aus dem Jahre 2009 [2], bei der insgesamt 57 Studien mit über 9000 Patienten analysiert wurden, waren die Erfolgsraten vergleichbar. Diese betrugen für AVNRT 94\%, für Vorhofflattern 92\% und für AVNRT kombiniert mit WPW 91\%. Diese Zahlen decken sich weitgehend mit unseren Ergebnissen mit akuten Erfolgsraten von je $100 \%$ bei AVNRT und isthmusabhängigem Vorhofflattern, von $89 \%$ bei WPW und $85 \%$ bei fokaler atrialer Tachykardie, was zeigt, dass die Ablation von SVT heute eine Routineeingriff mit beeindruckend hohem akuten Erfolg ist.

Die längerfristigen Erfolgsraten für supraventrikuläre Tachykardien sind gemäss mehreren Studien ebenfalls hoch. In einer früheren Zusammenstellung aus Zürich [9] betrug diese bei 243 konsekutiven Patienten 99\% nach sechs Monaten. In anderen Studien wurden Rezidivraten von 1,5\% für AVNRT [10] (bei 1419 Patienten über 63 Monate), von 4\% für WPW [11] (nach median 48 Monaten), von 10\% für fokale atriale Tachykardien [12] (bei 80 Patienten über 33 Monate) sowie von 11\% für typisches Vorhofflattern (in einer Meta-Analyse [13] mit über 10000 Patienten) beschrieben. Eine Analyse der Rezidivraten war nicht Teil unserer aktuellen Studie, sodass diesbezüglich keine Aussagen für unsere Studienpopulation getroffen werden können und hierfür weitere Untersuchungen erforderlich sind.

Fast die Hälfte aller Untersuchungen wurde ambulant durchgeführt; nur 7\% der Patienten blieben länger als eine Nacht im Spital. Diese Ergebnisse sind mit einer früheren Studie aus Frankreich und der französischen Schweiz [14] vergleichbar. In letzterer Studie mussten lediglich 0,6\% der Patienten aufgrund von Komplikationen im Bereich der Einstichstelle oder aufgrund von Lungenembolien rehospitalisiert werden.

\section{Komplikationen bei SVT Ablation}

Die Komplikationsraten lagen bei den oben zitierten, internationalen Studien je nach Rhythmusstörung zwischen 0,5 und 2,9\%, die Mortalität jeweils bei 0,02\% und damit sehr tief. Die häufigsten Komplikationen in der erwähnten Meta-Analyse [2] waren mit 1,4\% ein kompletter AV-Block mit Implantation eines Schrittmachers in 0,65\% aller Fälle, ein Hämatom in 0,3\% und ein Perikarderguss in 0,2\% der Ablationsbehandlungen. 
In der spanischen Registerstudie [8] wurden die Komplikationsraten noch differenzierter analysiert. Die meisten Komplikationen traten hier bei der Ablation von atypischem Vorhofflattern bzw. intraatrialen Reentry-Tachykardien auf. Von insgesamt 227 Ablationsbehandlungen kam es bei 5 Patienten zu einer relevanten Komplikation (2-mal Perikardtamponade, einmal kompletter AV-Block, einmal Gefässkomplikation und einmal akutes Lungenödem). Von insgesamt 333 Ablationsbehandlungen fokaler atrialer Tachykardien traten bei 2,1\% nennenswerte Komplikation auf, wobei 4 Patienten einen Perikarderguss entwickelten, zwei vaskuläre Komplikationen aufwiesen und bei einem aufgrund eines AV-Blocks ein Schrittmacher implantiert wurde. Die dritthäufigste Komplikationsrate lag mit 1\% ( $\mathrm{n}=25$ von 2485 ) bei typischem Vorhofflattern vor. Zwei Patienten erlitten einen Schlaganfall, bei einem erfolgte aufgrund eines AV-Blocks eine Schrittmacher-Implantation und bei je einem Patienten kam es zur Entwicklung eines Perikardergusses, zu einer kardialen Dekompensation und zu einer Lungenembolie. In dieser Gruppe waren zwei Todesfälle (Stroke und kardiogener Schock) zu verzeichnen. Von den 1999 Patienten, bei denen eine zusätzliche akzessorische Bahn (WPW) abladiert wurde, entwickelten 1,4\% eine relevante Komplikation mit 15 Gefässkomplikationen, 4 Schrittmacher-Implantationen infolge eines AVBlocks, 3 Schlaganfällen, 4 Herzinfarkten und 2 Perikardergüssen. Die geringste Komplikationsrate mit $0,6 \%$ ( $n=17$ von 2842 ) wurden bei Ablationsbehandlungen von AVNRTs beobachtet, wobei bei 0,35\% aufgrund eines AV-Blocks eine Schrittmacher-Implantation erforderlich wurde, bei 6 eine Gefässkomplikation und bei einem eine Lungenembolie auftrat.

Unsere Komplikationsraten waren mit 1,1\% ähnlich tief wie in den genannten Studien. In 2 Fällen $(1,1 \%$ der AVNRT-Ablationen) kam es zu einem kompletten AVBlock im Rahmen einer Ablation des slow pathways bei (typischer und atypischer) AVNRT wobei nur in einem Fall eine Schrittmacher-Implantation erforderlich wurde (0,54\% der AVNRT- bzw. 0,29\% aller SVT-Ablationen). Ein hämodynamisch nicht relevanter Perikarderguss, welcher keine therapeutischen Konsequenzen nach sich zog, trat im Rahmen einer Ablation eines linksseitigen WPW auf. Bei einem Patienten kam es nach Ablation eines isthmusabhängigen Vorhofflatterns zu einem relevanten Hämatom in der Leiste $(1,5 \%$ der typischen VHFla- bzw. 0,3\% aller SVT-Ablationen). Lungenembolien, kardiale Dekompensationen, Herzinfarkte oder Schlaganfälle sowie periinterventionelle Todesfälle traten in unserer Studienpopulation nicht auf.

\section{Schlussfolgerung}

Die Radiofrequenzablation hat sich für supraventrikuläre Tachykardien als äusserst erfolgreiches Behandlungsverfahren etabliert. Sie stellt für viele dieser Rhythmusstörungen heutzutage die Therapie der Wahl dar, zumal sie in vielen Fällen zu einer definitiven Heilung führt.

Die Häufigkeitsverteilung supraventrikulärer Tachykardien, der akute Ablationserfolg sowie die hiermit assoziierten Komplikationsraten sind in unserer Studie an einem tertiären Schweizer Zentrum weitgehend vergleichbar mit den internationalen Erfahrungen. Die Komplikationsrate ist insgesamt sehr gering, weswegen die Ablation bei einer Vielzahl der Patienten ambulant durchgeführt werden kann.

\section{Disclosure statement}

Die Autoren erhielten Forschungs- und Educational Grants für ihre Institution von Bayer Healthcare, Biosense Webster, Biotronik, Boston Scientific, Daiichi Sankyo, Medtronic, Actelion und St. Jude Medical. Thomas F. Lüscher: Beratungs- und/oder Vortragshonorare von AstraZeneca, Bayer Healthcare, Daiichi Sankyo und Medtronic. Jan Steffel: Beratungs- und/oder Vortragshonorare von Amgen, AstraZeneca, Atricure, Bayer, Biosense Webster, Biotronik, Boehringer-Ingelheim, Boston Scientific, Bristol-Myers Squibb, Cook Medical, Daiichi Sankyo, Medtronic, Novartis, Pfizer, Roche, Sanofi-Aventis, Sorin, St. Jude Medical und Zoll. Dr. Steffel ist Co-Director von CorXL. Alexander Breitenstein: Educational Grants von Biotronik, Biosense Webster und Actelion. Ardan M. Saguner: Vortragshonorar und Reiseunterstützung für wissenschaftliche Kongresse von Boston Scientific, Cambridge, MA und Biosense Webster, Diamond Bar, CA. Laurent Haegeli: Beratungs- und Vortragshonorare von St. Jude Medical. Die übrigen Autoren erklären keine potentiellen Interessenskonflikte.

\section{Referenzen}

Die vollständige Literaturliste finden Sie in der Online-Version des Artikels unter www.cardiovascmed.ch. 